\title{
Antipsychotics in the management of behavioral and psychological symptoms of dementia: maximizing gain and minimizing harm
}

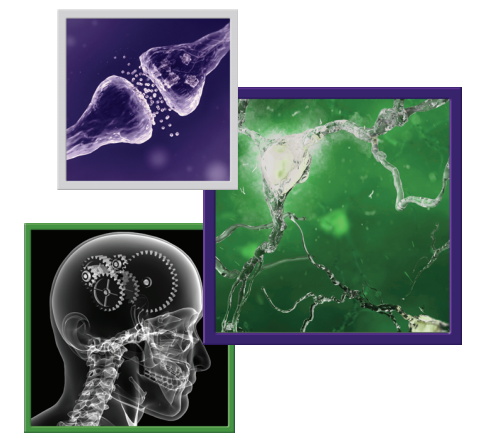

\author{
Rajesh R Tampi*,1,2 (D), Deena J Tampi ${ }^{3}$, Kathleen Rogers ${ }^{4}$ \& Suganthi Alagarsamy ${ }^{4}$ \\ ${ }^{1}$ Department of Psychiatry \& Behavioral Sciences, Cleveland Clinic Akron General, Akron, OH 44307, USA \\ ${ }^{2}$ Cleveland Clinic Lerner College of Medicine of Case Western Reserve University, Cleveland, OH 44195, USA \\ ${ }^{3}$ Diamond Healthcare, Richmond, VA 23219, USA \\ ${ }^{4}$ Department of Internal Medicine, Cleveland Clinic Akron General, Akron, OH 44307, USA \\ *Author for correspondence: Tel.: +1 203809 5223; Fax: +1 330344 2943; rajesh.tampi@gmail.com
}

\begin{abstract}
"Available data indicate that antipsychotic medications should be reserved for treating BPSD that are severe and/or refractory to nonpharmacological management strategies."
\end{abstract}

First draft submitted: 29 November 2019; Accepted for publication: 10 December 2019; Published online: 6 February 2020

Keywords: antipsychotics $\bullet$ behavioral and psychological symptoms of dementia $\bullet$ BPSD • cerebrovascular adverse events • meta-analysis $\bullet$ neuropsychiatric symptoms $\bullet$ systematic reviews

Behavioral and psychological symptoms of dementia (BPSD) include a wide array of noncognitive symptoms and behaviors that are seen in approximately $90 \%$ of individuals with dementia [1]. BPSD is associated with worse outcomes, including the risk for institutionalization and an increase in the overall cost of caring for individuals with dementia $[1,2]$.

Evidence indicates that both nonpharmacological and pharmacological treatments have shown benefit in the management of BPSD [3]. Among the nonpharmacological interventions, music therapy and behavioral management techniques were noted to be effective in reducing BPSD [4]. Pharmacotherapy is often used to treat BPSD that are nonresponsive to nonpharmacological interventions [5]. Medication classes that have been used in the management of BPSD include anticonvulsants, antidepressants, antipsychotics and cognitive enhancers [3]. Pharmacotherapy is often used after nonpharmacological interventions have failed, given their modest efficacy in managing BPSD and their significant adverse effect profile.

Available evidence indicates that antipsychotics are among the most commonly prescribed medication classes to treat BPSD, with approximately $27.5 \%$ of individuals with dementia being prescribed antipsychotics [6]. Greater prevalence of antipsychotic use was noted among individuals living at long-term care facilities and with greater severity of dementia possibly due to the greater prevalence of BPSD noted among individuals residing at these facilities.

In this editorial, we review the evidence for the use of antipsychotics in the management of BPSD from some important systematic reviews and meta-analyses that were published within the past 5 years.

\section{Efficacy of antipsychotics for management of BPSD}

Multiple studies have evaluated the efficacy of antipsychotics for the management of BPSD. Tampi et al., in their systematic review found 12 meta-analyses that evaluated the efficacy of antipsychotic medications in individuals with dementia [7]. Of these 12 studies, ten studies evaluated atypical antipsychotic medications, whereas two studies evaluated typical antipsychotics. The authors found that atypical antipsychotic medications, such as risperidone, olanzapine and aripiprazole, had modest efficacy in the management of BPSD. They also noted that quetiapine had limited efficacy when used in individuals with BPSD. The authors found that psychosis, aggression, agitation and more severe symptoms were typically responsive to the atypical antipsychotics. The two meta-analyses that 
evaluated the use of typical antipsychotics for BPSD found modest efficacy (effect size $=0.18$ in study one and odds ratio $[\mathrm{OR}]=2.81$ in study two) for these drugs in the management of BPSD, with noninferiority for any particular medication.

In another meta-analysis, Smeets et al., found that typical antipsychotics had a small effect on agitated patients on the agitation scales (standard mean deviation $[\mathrm{SMD}]=-0.44$ ) and for psychotic patients on the psychosis scales $(\mathrm{SMD}=-0.31)$, with no effects noted on the generic scales ( $\mathrm{SMD}=-0.00$ and -0.04 , respectively) [8]. The authors also found that they could not establish the efficacy of atypical antipsychotics for agitated patients on the agitation scales $(S M D=-0.15)$ and for psychotic patients on the psychosis scales (SMD $=-0.11$ ), with small effect size noted on mixed patients on agitation scales (SMD $=-0.29$ and -0.13 , respectively). Including studies with individuals having any BPSD symptom instead of specifically agitation, may have diluted the effect of typical antipsychotics on agitation.

In a network meta-analysis, Kongpakwattana et al., found that risperidone was superior to haloperidol and quetiapine for BPSD [9]. The authors did not find superior efficacy for haloperidol when compared with placebo for BPSD. In addition, haloperidol was found to be less efficacious when compared with all the medications in the network for BPSD.

\section{Adverse effects of antipsychotics when used among individuals with dementia}

Tampi et al., in their systematic review found that ten meta-analyses had evaluated the adverse effect profile of antipsychotics in the management of BPSD [7]. The authors found that antipsychotic use among individuals with BPSD was associated with a greater number of adverse effects when compared with placebo. These included risk for cerebrovascular adverse effects (CVAEs) and deaths. The authors found that the risk of CVAEs was most prominent among the risperidone-treated individuals when compared with placebo. They also discovered that the risk of death was not associated with any particular drug and only became apparent when the antipsychotic drugs were pooled together, as the number of deaths in each medication trial was small and the hazard ratio was not statistically significant. The authors noted that the risk of death did not correlate with the severity of dementia or behavioral symptoms, by the sample selection or by any particular diagnosis. They noted that sedation, abnormal gait and extrapyramidal symptoms were more common among the risperidone and olanzapine treated individuals.

Zhai et al., in their meta-analysis which included data from 12 studies, found that the pooled relative risk of death among individuals with dementia was 1.36 (95\% CI: 0.83-2.24) among antipsychotic drugs users versus nonusers [10]. The authors stated that when they excluded three studies that contributed to the significant heterogeneity in the study, the pooled relative risk for death increased to 2.08 (95\% CI: 1.39-3.13). In a metaanalysis by $\mathrm{Hsu}$ et al., which evaluated data from ten studies, the investigators found an increased risk for cerebrovascular accident (CVA) with first-generation antipsychotics $(\mathrm{OR}=1.49$; 95\% CI: 1.24-1.77), but not with second-generation antipsychotics $(\mathrm{OR}=1.31 ; 95 \% \mathrm{CI}$ : 0.74-2.30) [11]. They also found that the use of any antipsychotics among individuals with dementia was associated with a low risk of CVA (OR $=1.17 ; 95 \% \mathrm{CI}$ : 1.08-1.26). A meta-analysis by Rao et al., which evaluated data from population-based studies, found that the risk of CVA among individuals with dementia who were prescribed atypical antipsychotics was $1.61 \%$ when compared with $1.06 \%$ among individuals prescribed typical antipsychotics (relative risk $=1.02 ; \mathrm{p}=0.96$ ) [12].

In a meta-analysis by Wolf $e t$ al., which included data from ten studies, the authors found that there was no significant effect on cognition with the use of antipsychotics among individuals with dementia (SMD = -0.065), but they noted a substantial correlation between cognitive impairment and treatment duration $(\mathrm{p}<0.02)$ and also baseline cognition as measured by the Mini Mental State Examination $(\mathrm{p}<0.005)$ [13].

\section{Does withdrawal of antipsychotics improve outcomes among individuals with BPSD?}

Tampi et al., in their systematic review, found two meta-analyses that indicated that the discontinuation of antipsychotic medication may not worsen behavioral symptoms among all individuals with dementia, but individuals with greater baseline behavioral symptoms may have a worsening in their symptoms with antipsychotic discontinuation [7]. In one meta-analysis, the authors found that mortality rates were lower among the antipsychotic discontinuation group when compared with the antipsychotic continuation group.

In a meta-analysis that evaluated data from seven studies, Van Leeuwen et al., found that the discontinuation of antipsychotics made limited difference on whether or not individuals completed the study [14]. The investigator's intended primary outcome was the success of withdrawal of antipsychotics as defined by the ability of individuals to complete the study in the allocated study group (no failure because of worsening of BPSD or relapse to 
antipsychotic drug use). But this outcome was not reported in any of the included studies. Hence, they used the difference between groups in the number of noncompleters of the study as a proxy for the primary outcome. In two studies, in individuals with BPSD who had responded to antipsychotic treatment, the discontinuation of antipsychotics resulted in a greater number of individuals leaving the study prematurely because of relapse of symptoms. The investigators found that the discontinuation of antipsychotics made no difference to the overall severity of BPSD symptoms (group mean difference on the neuropsychiatric inventory score $=-1.49$ ). Two trials indicated that the discontinuation of antipsychotics may result in worsening of BPSD symptoms among individuals who had severe symptoms at baseline.

\section{When to use antipsychotics among individuals with BPSD?}

Available evidence indicates modest efficacy and multiple serious adverse effects, including CVAEs, possible cognitive decline and death when antipsychotics are used among individuals with BPSD [7,15]. The use of antipsychotics should be reserved for severe or refractory symptoms where nonpharmacological approaches or other medication classes have failed to produce the desired effect or are not feasible to use. The recommendation to use nonpharmacological approaches to initially manage BPSD is often to minimize the exposure of these individuals to pharmacotherapeutic agents especially the antipsychotics given their significant adverse effect profile [16].

It would also be prudent for clinicians to follow national guidelines like the recent American Psychiatric Association practice guideline when prescribing antipsychotics to individuals with BPSD [17]. The American Psychiatric Association guideline recommends a risk and benefit analysis prior to prescribing antipsychotics to individuals with BPSD. If this analysis favors the use of antipsychotics among individuals with BPSD, then treatment should be initiated at the lowest possible dose of an antipsychotic medication and titrated up to the minimum effective dose as tolerated. Additionally, if these individuals experience clinically significant adverse effects from the medication, the potential risks versus benefits of using the medication should be reevaluated. Furthermore, if there is no noticeable benefit from the medication following a 4-week trial at an adequate dose of the medication, then the medication should be tapered and discontinued. The guideline also recommends that when there is a positive response to the medication trial, an attempt to taper and withdraw the medication should be made within 4 months of initiation of treatment following discussions with the individual and/or their surrogate decision maker(s). The only reason why a taper would not be possible is if the individual has experienced a significant recurrence of symptoms with previous trials of tapering the medication. It is also recommended that while the medication taper is being attempted, the individual should be evaluated monthly for at least 4 months after the medication has been discontinued, to identify signs of recurrence of BPSD. The guideline also recommends that in the absence of delirium, haloperidol should not be used as a first-line agent for managing nonemergent BPSD. Individuals with BPSD should not receive long-acting injectable antipsychotic medications unless they have a comorbid chronic psychotic illness.

\section{Conclusion}

Available data indicate that antipsychotic medications should be reserved for treating BPSD that are severe and/or refractory to nonpharmacological management strategies. Additionally, they should be used with caution among these individuals, given their significant adverse effect profile. After completing a risk-benefit analysis, using the lowest effective doses of these medications for the shortest possible time, with adequate oversight will assist with maximizing gain and minimizing harm from these medications.

Financial \& competing interests disclosures

The authors have no relevant affiliations or financial involvement with any organization or entity with a financial interest in or financial conflict with the subject matter or materials discussed in the manuscript. This includes employment, consultancies, honoraria, stock ownership or options, expert testimony, grants or patents received or pending, or royalties.

No writing assistance was utilized in the production of this manuscript.

\section{References}

1. Feast A, Orrell M, Charlesworth G et al. Behavioral and psychological symptoms in dementia and the challenges for family carers: systematic review. Br. J. Psychiatry 208(5), 429-434 (2016).

2. Feast A, Moniz-Cook E, Stoner C et al. A systematic review of the relationship between behavioral and psychological symptoms (BPSD) and caregiver well-being. Int. Psychogeriatr. 28(11), 1761-1174 (2016). 
3. Gerlach LB, Kales HC. Managing behavioral and psychological symptoms of dementia. Psychiatr. Clin. North Am. 41(1), 127-139 (2018).

4. Abraha I, Rimland JM, Trotta FM et al. Systematic review of systematic reviews of non-pharmacological interventions to treat behavioral disturbances in older patients with dementia. The SENATOR-OnTop series. BMJ Open 7(3), e012759 (2017).

5. Bessey LJ, Walaszek A. Management of behavioral and psychological symptoms of dementia. Curr. Psychiatry Rep. 21(8), 66 (2019).

6. Kirkham J, Sherman C, Velkers C et al. Antipsychotic use in dementia. Can. J. Psychiatry 62(3), 170-181 (2017).

7. Tampi RR, Tampi DJ, Balachandran S et al. Antipsychotic use in dementia: a systematic review of benefits and risks from meta-analyses. Ther. Adv. Chronic Dis. 7(5), 229-245 (2016).

8. Smeets CHW, Zuidema SU, Hulshof TA et al. Efficacy of antipsychotics in dementia depended on the definition of patients and outcomes: a meta-epidemiological study. J. Clin. Epidemiol. 101, 17-27 (2018).

9. Kongpakwattana K, Sawangjit R, Tawankanjanachot I et al. Pharmacological treatments for alleviating agitation in dementia: a systematic review and network meta-analysis. Br. J. Clin. Pharmacol. 84(7), 1445-1456 (2018).

10. Zhai Y, Yin S, Zhang D. Association between antipsychotic drugs and mortality in older persons with Alzheimer's disease: a systematic review and meta-analysis. J. Alzheimers Dis. 52(2), 631-639 (2016).

11. Hsu WT, Esmaily-Fard A, Lai CC et al. Antipsychotics and the risk of cerebrovascular accident: a systematic review and meta-analysis of observational studies. J. Am. Med. Dir. Assoc. 18(8), 692-699 (2017).

12. Rao A, Suliman A, Story G et al. Meta-analysis of population-based studies comparing risk of cerebrovascular accident associated with first- and second-generation antipsychotic prescribing in dementia. Int. J. Methods Psychiatr. Res. 25(4), 289-298 (2016).

13. Wolf A, Leucht S, Pajonk FG. Do antipsychotics lead to cognitive impairment in dementia? A meta-analysis of randomised placebo-controlled trials. Eur. Arch. Psychiatry Clin. Neurosci. 267(3), 187-189 (2017).

14. Van Leeuwen E, Petrovic M, van Driel ML et al. Discontinuation of long-term antipsychotic drug use for behavioral and psychological symptoms in older adults aged 65 years and older with dementia. J. Am. Med. Dir. Assoc. 19(11), 1009-1014 (2018).

15. Dyer SM, Harrison SL, Laver K et al. An overview of systematic reviews of pharmacological and non-pharmacological interventions for the treatment of behavioral and psychological symptoms of dementia. Int. Psychogeriatr. 30(3), 295-309 (2018).

16. Caspar S, Davis ED, Douziech A et al. Nonpharmacological management of behavioral and psychological symptoms of dementia: what works, in what circumstances, and why? Innov. Aging 2(1), igy001 (2018).

17. Reus VI, Fochtmann LJ, Eyler AE et al. The American Psychiatric Association Practice Guideline on the use of antipsychotics to treat agitation or psychosis in patients with dementia. Am. J. Psychiatry 173(5), 543-546 (2016). 